\title{
Aplicación del analisis de redes sociales al estudio de la estructura y desarrollo de una red de blogs económicos políticos en Argentina
}

\author{
Sergio Gabriel Guerrero - Universidad de Buenos Aires - Grupo Antropocaos ${ }^{1}$
}

\begin{abstract}
Resumen
Los blogs o weblogs pueden ser definidos como páginas web localizadas en la Internet con muy poca edición interna, realizadas en base a plantillas predeterminadas, en general provistas de comentarios on line de los lectores y presentados en orden cronológico inverso, con enlaces a otras páginas web o recursos en línea. La mayoría de las veces son creados y administrados por individuos o a lo sumo por grupos reducidos de personas. La creación de vínculos entre los blogs, que podemos llamar sociales se produce a través de el establecimiento de hiperenlaces o referencias entre ellos, este conjunto de blogs y sus hiperenlaces se llama muchas veces bloglósfera.
\end{abstract}

En Argentina apareció sobre todo a partir de 2005 un conjunto de blogs que tienen como uno de sus fines el debate de la actualidad política y económica del país, estos blogs mantienen lazos entre sí conformando una verdadera red.

El objetivo de este trabajo es el mapeo de la estructura en dos momentos diferentes y del desarrollo de esta red aplicando, entre otras, las herramientas tradicionales del análisis de redes sociales y la comparación de dicha red en dos momentos diferentes además de reflexiónar sobre la validez, alcances y límites de estas herramientas para el trabajo con este tipo de redes.

Palabras clave: Blogs- Política- Economía- Red

\begin{abstract}
Blogs or weblogs can be defined as pages Web located in the Internet with very little internal edition, in general provided with commentaries on line of the readers and be displayed in inverse chronological sequence, with links to other pages Web or resources in line. In general they are created and administered by individuals or at the most by groups reduced of people. Ties between blogs are created through the establishment of hyperlinks or references among them, this set of blogs and their hyperlinks are often called bloglósfera.
\end{abstract}

In Argentina a set of blogs appeared mainly in 2005 or after. They have as aim the debate of the polític and economic present time of the country, these blogs maintains ties to each other conforming a true network.

The objective of this work is mapping the structure in two different moments and the development of this network applying, among others, the traditional tools of the analysis of social networks and the comparation between two moments of this network, besides to thinking on the validity and boundaries of social networks tools.

Key words: Blog - Politics - Economics - Network.

\footnotetext{
${ }^{1}$ Enviar correspondencia a: sergue@gmail.com
} 


\section{Una introducción a los blogs}

Los blogs, también conocidos como weblogs o bitácoras son un tipo especial de sitios web: son realizados en su mayoría con plantillas predeterminadas, en general provistas de comentarios on line de los lectores y presentados en orden cronológico inverso, con enlaces a otras páginas web o recursos en línea. Lo más común es construir los blogs y alojarlos por medio de servicios especializados que facilitan su diseño y proveen de su alojamiento gratuito².

Los blogs pueden cumplir múltiples funciones entre ellas las de diarios personales, tambien dar consejos técnicos, informaciones y discusiones sobre espectáculos y deportes, opiniones y comentarios sobre la actualidad política y económica etc. En general son creados y administrados por individuos o a lo sumo por grupos reducidos de personas. En muchos de los blogs no esta expuesto el nombre real del autor del posteo o de los comentarios a éstos, en su lugar se usan sobrenombres (nicks).

Sitios web con las carácterísticas que después se consideraron atributos de los blogs empezaron a aparecer entre 1995 y 1997, pero fue desde 1999 cuando se empezó a percibir el fenómeno, que en lo que va del siglo XXI se desarrolló de manera explosiva:

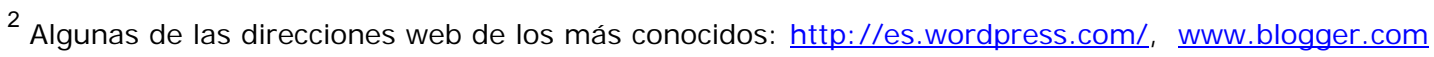




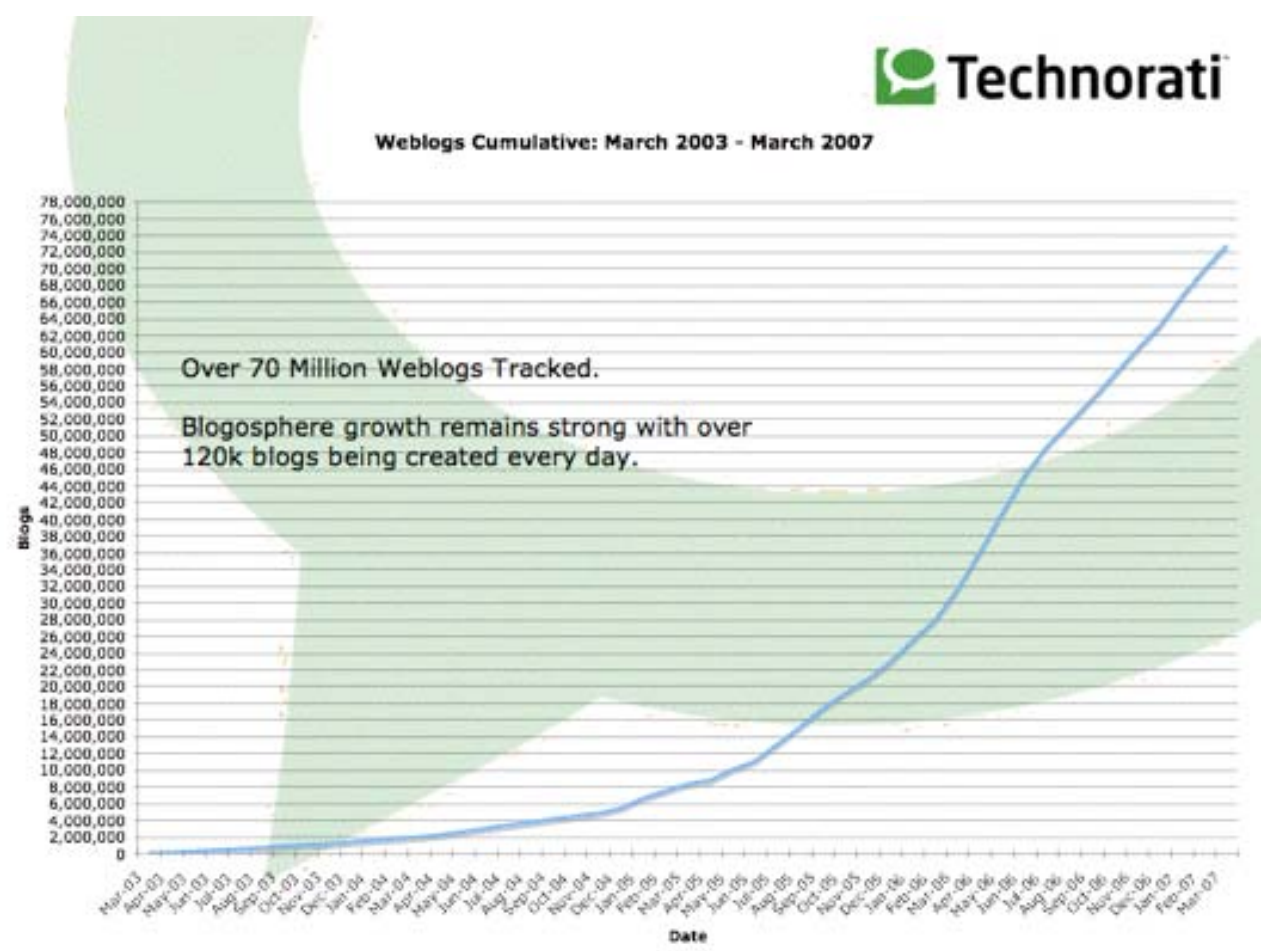

I lustración 1. Cantidad de blogs detectados por Technorati, uno de los principales buscadores de estos sitios $^{3}$.

Según Sifry (2007) la cantidad de blogs detectados por Technorati, un sitio web que es el principal motor de búsqueda de blogs pasó de dos millones en abril de 2004 a 10 millones en junio de 2005, calculándose en marzo de 2007, que son los últimos disponibles 72 millones. Según la misma fuente en marzo de 2007 alrededor del 3\% del movimiento relacionado con blogs se hace en idioma castellano.

Lo que resulta interesante, más allá del explosivo crecimiento de los blogs individuales es la aparición de nuevos tipos de relaciones sociales mediante el recurso técnico del hiperenlace entre diferentes blogs constituyendo la red o redes sociales, que se puede llamar blogósfera. La blogósfera no sería solamente el conjunto de blogs y de sus contenidos, sino también y sobre todo las conexiones e interacciones que existen entre ellos (Estatella, 2006).

Fuente Technorati en Sifry David: The State of the Live Web, April 2007 en http://www.sifry.com/alerts/archives/000493.html 


\section{Los blogs y lazos seleccionados}

En Argentina empezaron a surgir hace unos pocos años un conjunto de blogs que ponen su acento básicamente en debates y discusiones sobre la actualidad económica y política. Estas páginas poco a poco empezaron a tener más visibilidad en los medios de difusión tradicionales. También empezaron a tener entre ellas cada vez más enlaces y comentarios y debates cruzados con lo cual hoy se puede hablar con propiedad de la existencia de una de red de blogs políticos y económicos en Argentina.

Gran parte de este desarrollo se dió a partir de 2005. En nuestra selección de 67 blogs 4 fueron creados en 2003, 3 en 2004, 14 en 2005. 32 en 2006 y 14 en los primeros meses de 2007, o sea casi el 90\% fue creado a partir de 2005 .

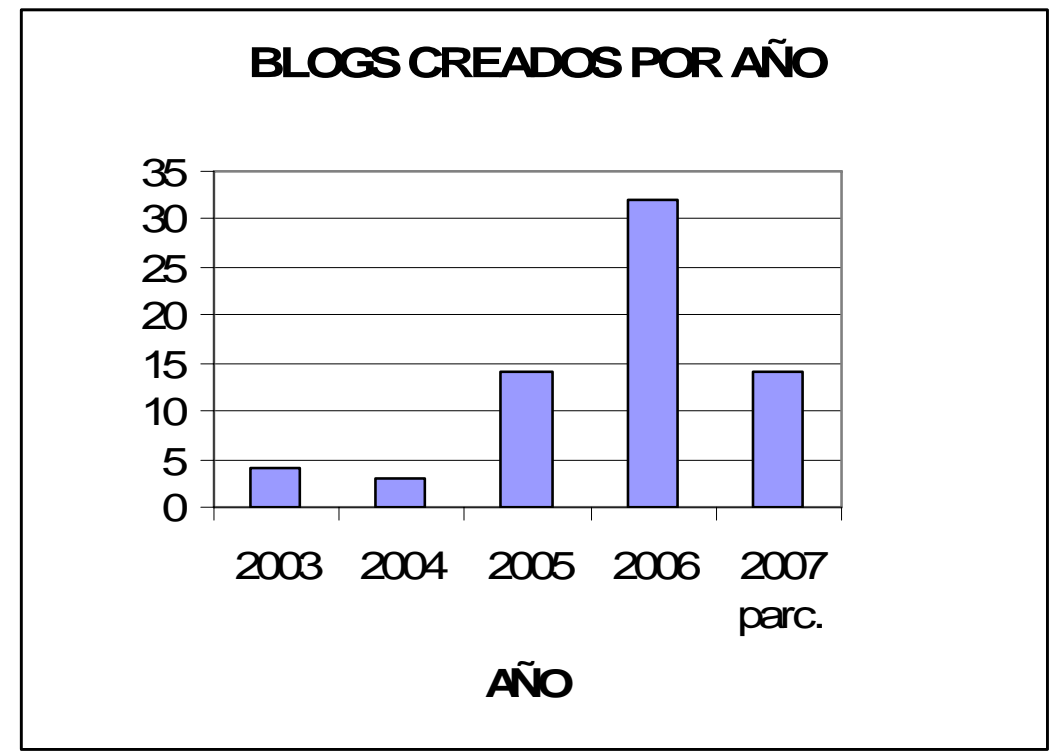

I lustración 2. Blocs creados por año.

Un problema que tuvimos que afrontar fue el de la manera de seleccionar nuesta muestra, la blogosfera no tiene límites claros ni tampoco es fácil definir sus diferentes partes o regiones. A nosotros nos interesaba mapear blogs argentinos que analizaran o comentaran temas de la actualidad económica o política nacional.

Comenzamos considerando como susceptibles de ser seleccionados sitios webs que cumplieran con las siguientes carácterísticas: 
1. Páginas que cumplan con el formato de Blogs, o sea que su parte principal esté compuesta por artículos ordenados en orden cronológico inverso.

2. Blogs activos, el criterio fue que hayan tenido artículos posteados en junio o julio de 2007.

3. Que una parte significativa de los artículos traten sobre temas de la actualidad económica o política de Argentina.

4. Que esté abierta la opción de comentarios, o sea que los lectores tengan la posibilidad de dejar sus comentarios a continuación de los artículos.

Para seleccionar los blogs con los que íbamos a trabajar utilizamos una variante de lo que se conoce como "muestreo en bola de nieve". Comenzamos con 9 sitios que conocíamos y que cumplían dichas condiciones, en lo que conocemos como "etapa 0" a partir de esos 9 blogs, comenzamos a explorar los vínculos presentes en sus blogrolls, si esos vínculos nos llevaban a páginas que cumplían con las características buscadas, los agregámos a nuestra muestra, en la "etapa 1". Finalmente hicimos lo mismo con los blogs encontrados en esta zona y agregamos los blogs encontrados en una "etapa 2". Nuestra muestra quedó contruida por 9 blogs de la etapa 0, 25 de la zona de la etapa 1 y 33 en la etapa 2, en total 67 blogs.

Este tipo de muestreo es originado en una idea de Goodman (1961), y es muy útil para el mapeado de redes de cualquier tipo, sobre todo cuando los límites de la red no se conocen o no están claramente definidos ya que permite considerar no sólo los nodos, sino también los lazos o relaciones que los vinculan, quedarían fuera de la muestra nodos no vinculados directa o indirectamente con los nodos de origen 0 semillas (etapa 0), pero este problema se puede minimizar aumentando el número de semillas, que es lo que nosotros hicimos.

Una vez que tuvimos definida nuestra muestra relevamos los lazos que tenían los diferentes nodos entre sí:

En general podemos considerar 3 tipos de lazos como indicadores de relación entre blogs. 
1. Lo que se conoce como vinculaciones a través de los blogrolls, son hiperenlaces que se encuentran en general agrupados en una columna a la izquierda o derecha de la pantalla a la página de inicio de otros blogs.

2. Los que se conocen como permalink, son hiperenlaces a artículos específicos en otros blogs, que se encuentran en general en el cuerpo de los artìculos del blog.

3. Los comentarios, se encuentran después de cada artìculo y pueden servir también para establecer vinculaciones entre blogs, los comentarios en general no son hechos por los creadores del blog en cuestión, aunque pueden hacerlos como parte de una discusión o debate que se desarrolle allí.

En esta primera etapa tomamos los vínculos que figuran en los blogrolls, estos en general son los más estables ya que dependen de decisiones de los creadores de los blogs ponerlos o sacarlos. Nuestra idea es en etapas siguientes trabajar con los permalinks y los vínculos generados por comentarios para poder establecer el desarrollo y los cambios en esta red.

Así elaboramos la matriz de relaciones entre blogs. Es una matriz de un modo, cuadrada, binaria, o sea sólo relevamos la presencia o ausencia del lazo y no su fuerza relativa o absoluta y no simétrica (que $\mathbf{A}$ se relacione con $\mathbf{B}$ no implica necesariamente que $\mathbf{B}$ se relacione con $\mathbf{A}$ ).

Por otro lado también recogimos ciertos atributos de nuestros Blogs, de ellos seleccionamos dos, el indice de autoridad de Technorati ${ }^{4}$, este índice se construye tomando la cantidad de blogs "alter"que se refirieron al blog "ego" en los ùltimos 6 meses y, finalmente, el mes y año de creación de cada blog perteneciente a nuestra muestra:

En una segunda etapa realizada durante junio y julio de 2008, elaboramos una segunda matriz de relaciones entre los mismos blogs y con los mismos criterios, pero con los enlaces presentes en los blogrolls en junio-julio de 2008.

\footnotetext{
${ }^{4}$ http://support.technorati.com/faq/topic/ 71
} 


\begin{tabular}{|c|c|c|c|c|c|}
\hline Cod & Nombre & Dirección Web & $\begin{array}{c}\text { Índice } \\
\text { autoridad } \\
\text { Technorati } \\
2007 \\
\end{array}$ & $\begin{array}{c}\text { Indice } \\
\text { Autoridad } \\
\text { Techorati } 2008\end{array}$ & $\begin{array}{c}\text { Año-mes } \\
\text { Inicio } \\
\text { Blog }\end{array}$ \\
\hline LCM & La ciencia maldita & http://lacienciamaldita.blogspot.com/ & 42 & 42 & 2005-06- \\
\hline RAT & Rambe Tamble & http://rambletamble.blogspot.com/ & 137 & 122 & 2006-01- \\
\hline HEC & Homo Economicus & http://www.homo-economicus.blogspot.com/ & 32 & 85 & 2006-03- \\
\hline OLI & Olivera & http://wwww.olivera.blogspot.com/ & 28 & 18 & 2003-04- \\
\hline $3 \mathrm{CH}$ & Tres Chiflados & http://los3chiflados.blogspot.com/ & 24 & 35 & 2006-06- \\
\hline ABE & Abuelo Economico & http://www.abueloeconomico.blogspot.com/ & 43 & 37 & 2006-06- \\
\hline CRG & Criador de Gorilas & http://www.elcriadordegorilas.blogspot.com/ & 53 & 79 & 2006-04- \\
\hline LBA & La Barbarie & http://labarbarie.com.ar/ & 112 & 276 & 2005-10- \\
\hline $\mathrm{AVI}$ & Avila & http://www.jorgeavilaopina.com/ & 19 & 13 & 2006-02- \\
\hline CRE & Cron al de Reagan & http://ccronaldreagan.blogspot.com/ & 13 & 3 & 2006-01- \\
\hline ILU & lluminatios & http://www.iluminatios.blogspot.com/ & 3 & 3 & 2005-09- \\
\hline $50^{\mathrm{a}}$ & 50 amaneceres & http://www.50amaneceres.blogspot.com/ & 0 & 0 & 2006-06- \\
\hline MUP & Mundo Perverso & http://mundo-perverso.blogspot.com/ & 21 & 100 & 2003-12- \\
\hline CAR & Carnotistas & http://carnotistas.blogspot.com/ & 35 & 31 & 2005-12- \\
\hline ELE & El lobo estepario & http://el-lobo-estepario.blogspot.com/ & 0 & 0 & 2006-05- \\
\hline MNM & Mide no mide & http://vidabinaria.blogspot.com/ & 30 & 67 & 2006-06- \\
\hline DDM & Destructor de mitos & http://www.destructordemitos.com.ar/ & 0 & 16 & 2007-03- \\
\hline GCO & Grupo Concordia & http://www.grupoconcordia.blogspot.com/ & 14 & 4 & 2006-08- \\
\hline NPR & Nerd-Progre & http://nerd-progre.blogspot.com/ & 11 & 24 & 2005-04- \\
\hline$A D C$ & Alternativas de un cambio & http://alternativas-de-un-cambio.blogspot.com/ & 21 & 13 & 2006-05- \\
\hline ELI & El impreciso & http://el-impreciso.blogspot.com/ & 30 & 3 & 2006-10- \\
\hline $\mathrm{DHI}$ & Deshonestidad intelectual & http://deshonestidadintelectual.blogspot.com/ & 1 & 1 & 2006-12- \\
\hline DMR & Del medio recargado & http://delmediorecargado.blogspot.com/ & 6 & 6 & 2007-02- \\
\hline EBR & El Blog rayado & http://blograyado.blogspot.com/ & 14 & 6 & 2003-11- \\
\hline LCA & Lucas Carrasco & http://lucascarrasco.blogspot.com/ & 0 & 39 & 2006-11- \\
\hline $\mathrm{PCH}$ & Patria Chacarera & http://patriachacarera.blogspot.com/ & 12 & 44 & 2006-11- \\
\hline FDS & Faro del Sur & http://farodelsur.blogspot.com/ & 5 & 5 & 2007-05- \\
\hline TDM & Trade \& Me & http://tradeandme.blogspot.com/ & 18 & 4 & 2006-08- \\
\hline ESC & Economista Serial Crónico & http://econserialcronico.blogspot.com/ & 22 & 36 & 2006-09- \\
\hline EOJ & Econojota & http://econojota.blogspot.com/ & 5 & 0 & 2007-04- \\
\hline FIP & Finanzas públicas & http://musgrave-finanzaspublicas.blogspot.com/ & 6 & 31 & 2007-04- \\
\hline MAV & Marvakris y valdes & http://mavrakisyvaldes.blogspot.com/ & 24 & 13 & 2005-08- \\
\hline IAQ & Infrecuented A. Question & http://iaq4all.blogspot.com/index.html & 6 & 0 & 2006-12- \\
\hline LRP & La res publica & http://la-res-publica.com.ar/ & 31 & 21 & 2006-09- \\
\hline RTL & Revolucion tinta y limón & http://www.revolucion-tinta-limon.blogspot.com/ & 13 & 13 & 2006-04- \\
\hline MIF & Moreno y Fabianesi & http://morenoyfabianesi.blogspot.com/ & 2 & 2 & 2006-12- \\
\hline EBS & El Buen salvaje & http://www.elbuensalvaje.blogspot.com/ & 22 & 33 & 2005-11- \\
\hline SMR & Sine Metu Reloaed & http://sine-metu.blogspot.com/ & 34 & 17 & 2005-01- \\
\hline TIR & Tintas rojas & http://tintasrojas.blogspot.com/ & 8 & 9 & 2006-11- \\
\hline INC & Inconciente Colectivo & http://elinconscientecolectivo.blogspot.com/ & 21 & 0 & 2006-06- \\
\hline PVV & Periodismo viceversa & http://periodismoviceversa.wordpress.com/ & 1 & 1 & 2007-04- \\
\hline EDO & El Dotor & http://eldotor.blogspot.com/ & 4 & 10 & 2006-08- \\
\hline A3P & A tres puntas & http://atrespuntas.blogspot.com/ & 14 & 8 & 2007-04- \\
\hline MEG & Multitud ególatra & http://multitudegolatra.blogspot.com/ & 27 & 32 & 2006-08- \\
\hline LMA & Periodístico La Maga & http://lamagaprensa.blogspot.com/ & 1 & 5 & 2007-05- \\
\hline EAG & El Aeronauta & http://weblogs.clarin.com/el-agronauta/ & 0 & 7 & 2007-06- \\
\hline MDG & El extraño mundo de D.Goldman & http://diegogoldman.blogspot.com/ & 7 & 7 & 2005-09- \\
\hline EOC & El opinador compulsivo & http://articulos-interesantes.blogspot.com/ & 25 & 52 & 2004-09- \\
\hline JSK & JSK_SDE & http://jsk-sde.blogspot.com/ & 13 & 12 & 2003-03- \\
\hline MKT & MarcosKtulu & http://marcosktulu.blogspot.com/ & 21 & 26 & 2005-01- \\
\hline CSR & Un camino sin retorno & http://prendanlaluz.blogspot.com/ & 5 & 6 & 2006-02- \\
\hline $\mathrm{BBI}$ & Blogbis & http://blogbis.blogspot.com/ & 54 & 72 & 2004-12- \\
\hline LIC & Liberticracia & http://liberticracia.blogspot.com/ & 28 & 16 & 2006-11- \\
\hline DIP & Division Política & http://www.divisionpolitica.com.ar/ & 3 & 3 & 2007-03- \\
\hline JOT & Jorge Telerman & http://jtelerman.blogspot.com/ & 179 & 13 & 2007-03- \\
\hline EFC & El fondo de la cuestión & http://elfondodelacuestion.blogspot.com/ & 1 & 2 & 2007-06- \\
\hline BIN & El Blog del Ingeniero & http://desdegambier.blogspot.com/ & 0 & 16 & 2007-06- \\
\hline EDK & Educadores & http://edukadores.blogspot.com/ & 5 & 2 & 2006-06- \\
\hline LSP & Los sepultureros del pueblo & http://sepultureros.blogspot.com/ & 3 & 0 & 2007-03- \\
\hline
\end{tabular}




\begin{tabular}{lllrr} 
CIU & El ciudadano & http://elciudadanoargentino.blogspot.com/ & 12 & 14 \\
NCS & No cualquiera suma sin restar & http://juancruz-rgl.blogspot.com/ & $2005-07-$ & 52 \\
EHE & El hermeneuta & http://www.predicadOr.blogspot.com/ & 5 & $2005-05-$ \\
EXT & Excluidos pero Tecnologizados & http://excluidosperotecnologizados.blogspot.com/ & $2004-12-$ & 9 \\
QNP & Que nos parió & http://www.quenospario.blogspot.com/ & 3 & $2006-05-$ \\
DEN & Desarrollo Entrerriano & http://desarrolloentrerriano.wordpress.com/ & 1 & 1 \\
JDL & Jugo de Ladrillo & http://jugodeladrillo.blogspot.com/ & 1 & $2006-04-$ \\
CUS & ComentarlUS & http://iusandlaw.blogspot.com/ & 22 & 16 \\
\hline
\end{tabular}

Tabla 1. Tabla de Blogs seleccionados.

\section{Análisis}

Encontramos en nuestra búsqueda varios trabajos que utilizaban elementos de Análisis de Redes Sociales al estudio de los Blogs (Cameron 2004), (Herring et al 2005), (Kumar et al 2003), (Merelo et al 2004), (Shirky 2003), pero todos ellos en general trabajaban de manera más o menos automatizada con bases de datos muy grandes de blogs y lo que hacen sobre todo es ver qué tipo de distribución de lazos tienen y como éstas van evolucionando. Sólo en el trabajo de Herring encontramos algún uso de grafos y de herramientas de búsqueda de subgrupos.

Nosotros construimos nuestra muestra de manera manual y teniendo en cuenta entre otras cosas el contenido semántico de los artículos de blogs además de sus lazos, esto nos permite utilizar otras herramientas de análisis que las que utilizaron los autores citados hasta este momento, aunque con una base de datos con muchos menos registros.

\section{Datos Generales}

Tomando como nodos los diferentes blogs y como relaciones la presencia de hiperenlaces en los blogrolls teníamos en 2007 una red con una presencia de 67 nodos y 440 lazos lo que da una densidad de 0,0995. O sea, están presentes casi el $10 \%$ de los lazos posibles. En promedio cada nodo presentaba 6,57 lazos de entrada y salida pero con una gran variación entre diferentes nodos (desvío estándar lazos de entrada 6,13 y lazos de salida 5,88). La red solo tenía un componente, todos los nodos están directa o indirectamente conectados entre sí aunque algunos de ellos reciben lazos pero no los emiten, por lo que se puede caracterizar dicho componente como débil (no es posible siguiendo la dirección de los enlaces llegar desde cualquier nodo a cualquier otro). 
Los datos del 2008 son similares a los de 2007, aunque la red es ahora algo más densa y algo mas desconectada. Los mismos 67 nodos presentan 490 lazos con una densidad de 0,1108 , están presentes algo más del $11 \%$ de los lazos posibles. El promedio de lazos de entrada y de salida de cada nodo es de 7,31 con una gran variación entre diferentes nodos (desvío estándar lazos de entrada 7,14 y lazos de salida 6,08). La red presenta 5 componentes, 4 de ellos son nodos aislados y el otro reúne a 63 nodos que están directa o indirectamente conectados entre sí aunque algunos de ellos reciben lazos pero no los emiten o viceversa.

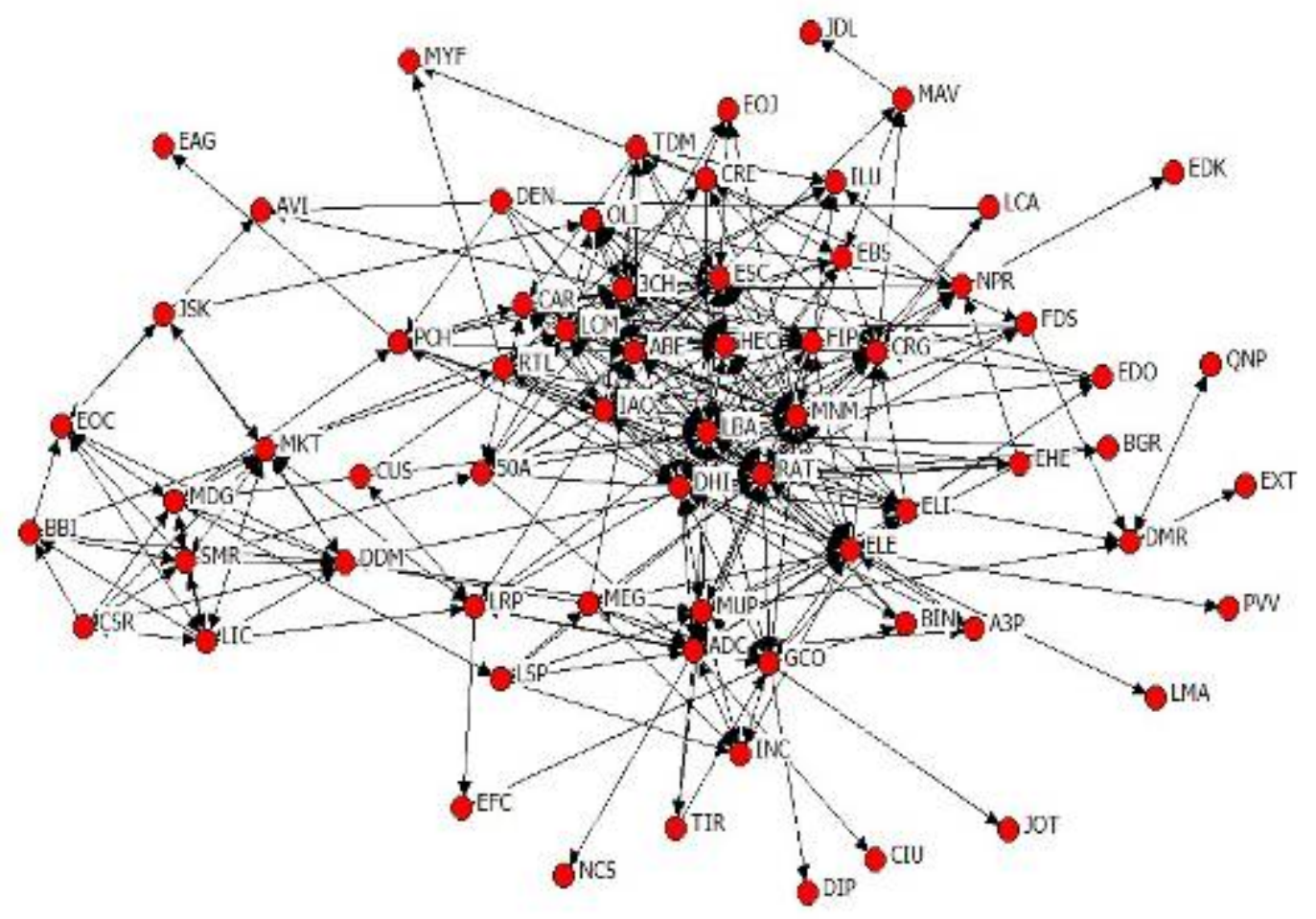

I lustración 3. Grafo con los diferentes blogs seleccionados representados como nodos y sus hiperenlaces en sus respectivos blogrolls presentes en 2007 como lazos. 
I lustración 4. Grafo con los diferentes blogs seleccionados representados como nodos y sus hiperenlaces en sus respectivos blogrolls presentes en 2008 como lazos.

\section{Medidas de Centralidad}

En la siguiente tabla presentamos las medidas de centralidad más utilizadas (grado, cercanía e intermediación en sus variantes normalizadas y simetrizadas

This routine automatically symmetrizes and binarizes. Normalized Centrality Measures

\begin{tabular}{rlrrr} 
& & \multicolumn{1}{c}{$\begin{array}{c}2 \\
\text { Degree }\end{array}$} & \multicolumn{1}{c}{$\begin{array}{c}3 \\
\text { Closeness }\end{array}$} & Betweenness \\
1 & LCM & 30.303 & 51.563 & 4.711 \\
2 & RAT & 43.939 & 63.462 & 19.757 \\
3 & HEC & 22.727 & 49.254 & 0.868 \\
4 & OLI & 15.152 & 46.154 & 1.661 \\
5 & 3 CH & 30.303 & 51.563 & 4.211 \\
6 & ABE & 27.273 & 50.769 & 3.934 \\
7 & CRG & 27.273 & 50.382 & 3.929 \\
8 & LBA & 42.424 & 58.929 & 10.479 \\
9 & AVI & 4.545 & 36.872 & 0.571 \\
10 & CRE & 10.606 & 42.308 & 0.940 \\
11 & ILU & 9.091 & 41.772 & 0.141 \\
12 & 5 ONA & 16.667 & 48.175 & 2.212 \\
13 & MUP & 19.697 & 51.969 & 4.766 \\
14 & CAR & 18.182 & 46.809 & 0.311 \\
15 & ELE & 30.303 & 52.800 & 8.796 \\
16 & MNM & 36.364 & 55.932 & 6.101 \\
17 & DDM & 16.667 & 45.833 & 6.610 \\
18 & GCO & 24.242 & 50.000 & 9.219 \\
19 & NPR & 12.121 & 44.898 & 3.256 \\
20 & ADC & 25.758 & 51.163 & 8.398 \\
21 & ELI & 18.182 & 48.889 & 0.774 \\
22 & DHI & 36.364 & 56.897 & 6.279
\end{tabular}




\begin{tabular}{lllll}
23 & DMR & 7.576 & 41.509 & 6.042 \\
24 & BGR & 3.030 & 41.250 & 0.000 \\
25 & LCA & 4.545 & 41.250 & 0.054 \\
26 & PCH & 15.152 & 47.482 & 4.355 \\
27 & FDS & 9.091 & 44.295 & 0.824 \\
28 & TDM & 13.636 & 40.994 & 0.211 \\
29 & ESC & 25.758 & 47.482 & 1.468 \\
30 & EOJ & 6.061 & 39.053 & 0.000 \\
31 & FIP & 22.727 & 48.889 & 0.605 \\
32 & MAV & 6.061 & 36.872 & 3.046 \\
33 & IAQ & 27.273 & 51.563 & 2.113 \\
34 & LRP & 13.636 & 46.809 & 3.619 \\
35 & RTL & 13.636 & 48.175 & 3.123 \\
36 & MYF & 3.030 & 33.333 & 0.000 \\
37 & EBS & 12.121 & 45.205 & 1.093 \\
38 & SMR & 12.121 & 38.824 & 0.863 \\
39 & TIR & 4.545 & 36.667 & 0.000 \\
40 & INC & 9.091 & 40.741 & 0.095 \\
41 & PVV & 1.515 & 34.737 & 0.000 \\
42 & EDO & 7.576 & 40.491 & 0.026 \\
43 & A3P & 6.061 & 40.994 & 0.000 \\
44 & MEG & 12.121 & 48.889 & 1.356 \\
45 & LMA & 1.515 & 34.737 & 0.000 \\
46 & EAG & 1.515 & 32.353 & 0.000 \\
47 & MDG & 13.636 & 39.521 & 1.881 \\
48 & EOC & 10.606 & 35.294 & 0.663 \\
49 & JSK & 6.061 & 36.264 & 0.354 \\
50 & MKT & 15.152 & 43.421 & 3.435 \\
51 & CSR & 9.091 & 34.921 & 0.031 \\
52 & BBI & 9.091 & 34.375 & 0.053 \\
53 & LIC & 12.121 & 37.931 & 0.676 \\
54 & DIP & 1.515 & 33.503 & 0.000 \\
55 & JOT & 1.515 & 33.503 & 0.000 \\
56 & EFC & 3.030 & 35.676 & 0.000 \\
57 & BIN & 6.061 & 43.421 & 0.000 \\
58 & EDK & 1.515 & 31.132 & 0.000 \\
59 & LSP & 9.091 & 44.000 & 0.752 \\
60 & CIU & 1.515 & 34.021 & 0.000 \\
61 & NCS & 1.515 & 34.021 & 0.000 \\
62 & EHE & 7.576 & 43.709 & 0.092 \\
63 & EXT & 1.515 & 29.464 & 0.000 \\
64 & QNP & 1.515 & 29.464 & 0.000 \\
65 & DEN & 10.606 & 43.709 & 0.826 \\
66 & JDL & 1.515 & 27.049 & 0.343 \\
67 & CUS & 6.061 & 42.581 & \\
6 & & & & \\
\hline
\end{tabular}

Tabla 2. Tabla de medidas de centralidad Normalizadas 2007.

Se puede ver que, mas allá de las covariaciones entre éstas medidas, en algunos casos el grado (degree) y la intermediación (betweeness) siguen tendencias distintas. Es posible que haya blogs que cumplan funciones de broker (intermediarios) entre diferentes sectores de la red, pese a que no se destaquen por la cantidad de enlaces que envían o reciben. Los casos mas notables son los siguientes que están bastante mejor situados en el ranking según los valores de intermediación que en el ranking según grado: GCO 3 y 13, DDM 6 y 20 y DMR 9 y 42.

This routine automatically symmetrizes and binarizes. Normalized Centrality Measures

$\begin{array}{cccrr} & & 1 & 2 & 3 \\ & & \text { Degree } & \text { Closeness } & \text { Betweenness } \\ 1 & \text { LCM } & 30.303 & 17.098 & 3.448 \\ 2 & \text { RAT } & 46.970 & 18.132 & 13.098 \\ 3 & \text { HEC } & 28.788 & 17.054 & 1.919 \\ 4 & \text { OLI } & 13.636 & 16.500 & 1.295 \\ 5 & 3 \mathrm{CH} & 31.818 & 17.188 & 4.389 \\ 6 & \text { ABE } & 33.333 & 17.323 & 3.396 \\ 7 & \text { CRG } & 34.848 & 17.277 & 3.395 \\ 8 \text { LBA } & 43.939 & 17.886 & 7.149 \\ 9 \text { AVI } & 4.545 & 15.278 & 0.913 \\ 10 & \text { CRE } & 4.545 & 15.313 & 0.024 \\ 11 & \text { ILU } & 13.636 & 16.058 & 0.114\end{array}$




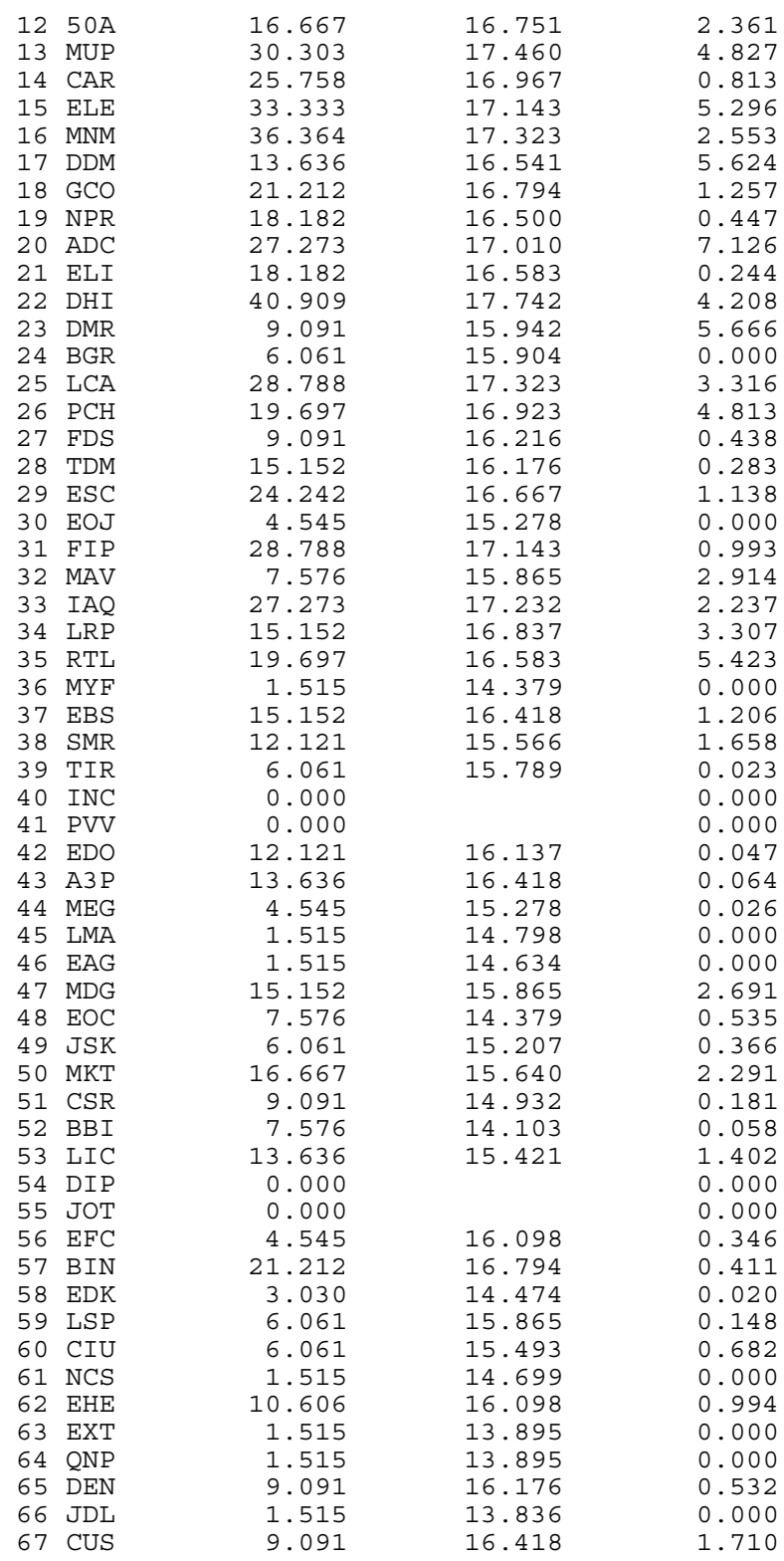

Important note: This network is disconnected. Technically, closeness should not be computed.

Tabla 3. Tabla de medidas de centralidad Normalizadas 2008.

En los datos de 2008 los nodos que más se destacan por cumplir esta función de intermediación aunque no tengan un número importante de enlaces son, con sus respectivas posiciones en ambos rankings DDM 5 y 31, DMR 4 y 38, ADC 3 y 14 , RTL 6 y 20 y MAV 17 y 43.

\section{Centro - Periferia}

Las relaciones entres los blogs seleccionados permiten separar de manera clara los que ocupan una posición central en la red estudianda y los que ocupan una posición periférica. Si aplicamos el algoritmo de Modelo de Núcleo Periferia en su opción 
Dicotómica, con la medida de adecuación Corr. A los datos de 2007, nos da como resultado la siguiente separación:

Núcleo: LCM RAT HEC OLI 3CH ABE CRG LBA 50A MUP CAR ELE MNM GCO ADC ELI DHI PCH ESC FIP IAQ

Periferia: AVI CRE ILU DDM NPR DMR BGR LCA FDS TDM EOJ MAV LRP RTL MYF EBS SMR TIR INC PVV EDO A3P MEG LMA EAG MDG EOC JSK MKT CSR BBI LIC DIP JOT EFC BIN EDK LSP CIU NCS EHE EXT QNP DEN JDL CUS

La región de la matriz que relaciona a los blog del núcleo entre sí tiene una densidad de 0,51 , las regiones que relacionan los nodos del núcleo con los de la periferia tiene una densidad de 0,074 y finalmente la región que relaciona los blogs de la periferia entre sí tiene una densidad de sólo 0,041. Los blogs del núcleo tienen en las relaciones entre ellos una densidad más de 12 veces mayor que los de la periferia:

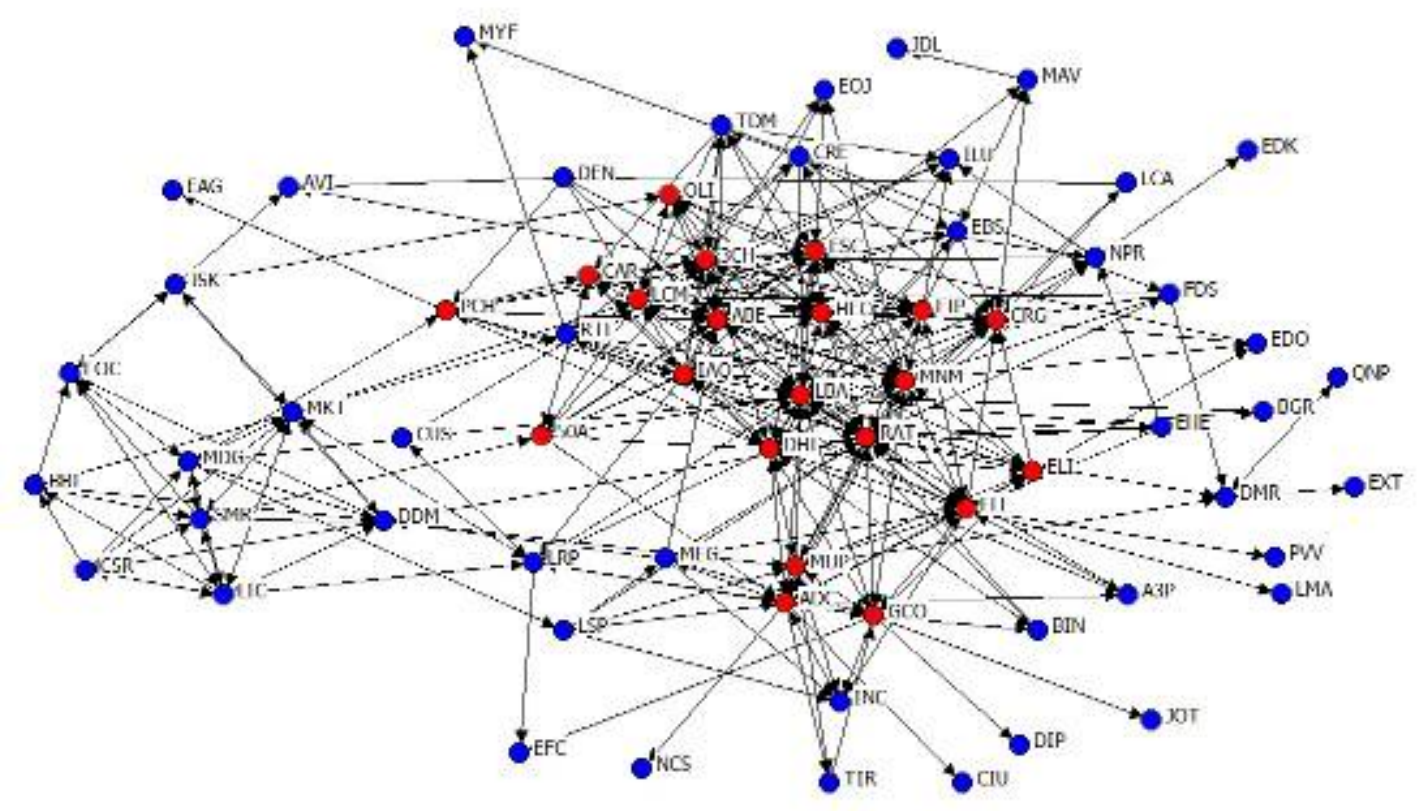

Ilustración 5. 2007. Rojo: Núcleo; Azul: Periferia

Si lo relacionamos con los atributos del ìndice de autoridad según technorati, vemos que las que están en el núcleo tienen un valor medio de 32 y en los de la periferia es de 16, lo que nos muestra una correlación bastante intensa entre nuestra 
selección de Blogs y la blogósfera total en relación con la centralidad de los blogs teniendo en cuenta los datos de 2007.

Esperábamos que los blogs mas centrales, del núcleo, presentaran una antigüedad visiblemente mayor que los de la periferia, y que ello explique en parte su mayor conectividad, pero los datos no muestra esto. El promedio de antigüedad de los blogs del núcleo en 2007 es de 16 meses y el de los de la periferia es de 15, no creemos que esta diferencia de un mes explique la diferencia de conexiones entre ellos.

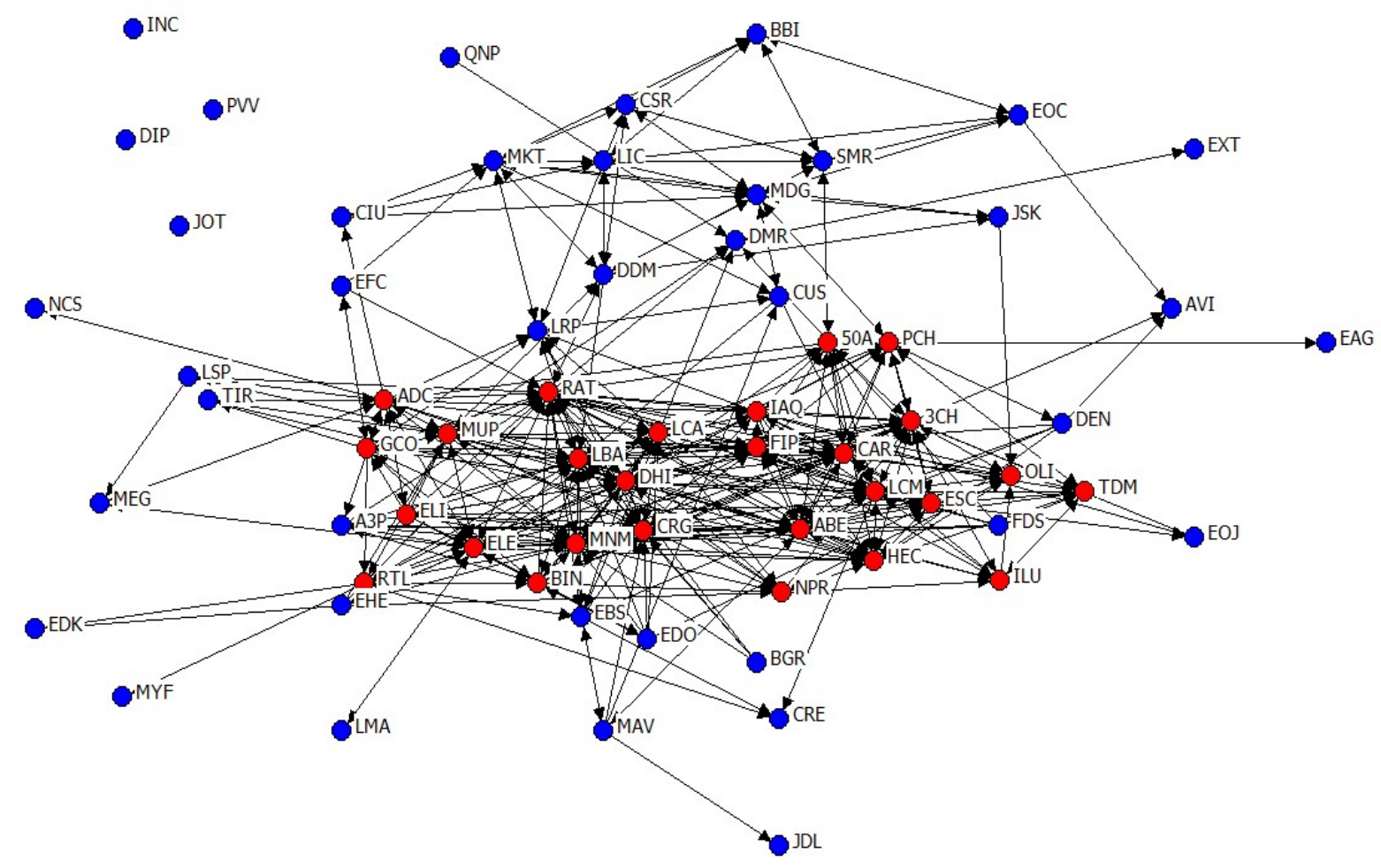

I lustración 6. 2008. Rojo: Núcleo; Azul: Periferia.

Si aplicamos el algoritmo de Modelo de Núcleo Periferia en su opción Dicotómica, con la medida de adecuación Corr. a los datos de 2008, nos da como resultado la siguiente separación:

Núcleo: LCM RAT HEC OLI 3CH ABE CRG LBA ILU 50A MUP CAR ELE MNM GCO NPR ADC ELI DHI LCA PCH TDM ESC FIP IAQ RTL BIN

Periferia: AVI CRE DDM DMR BGR FDS EOJ MAV LRP MYF EBS SMR TIR INC PVV EDO A3P MEG LMA EAG MDG EOC JSK MKT CSR BBI LIC DIP JOT EFC EDK LSP CIU NCS EHE EXT QNP DEN JDL CUS 
La región de la matriz que relaciona a los blog del núcleo entre sí tiene una densidad de 0,43 , las regiones que relacionan los nodos del núcleo con los de la periferia tiene una densidad de 0,053 y finalmente la región que relaciona los blogs de la periferia entre sí tiene una densidad de sólo 0,047. Los blogs del núcleo tienen en las relaciones entre ellos una densidad más de 9 veces mayor que los de la periferia, menor que la de 2007 pero con un núcleo bastante más extenso.

Con los datos de 2008 la correlación entre la la pertenencia al núcleo o periferia de nuestra red y los datos del Ranking de Technorati es todavía mayor que en 2007 los blogs del núcleo tienen un valor medio de 42 y entre los blogs de la pericia dicho valor cae a 13 .

A pesar de que no encontramos con los datos de 2007 y aún menos con los de 2008 una diferencia significativa entre el núcleo y la periferia en relación con la antigüedad promedio de los blogs como vemos mas abajo, la distribución de lazos entrantes sigue un patrón que se asemeja al de las redes libres de escala tanto para los datos de 2007 como para los del 2008, en el cual deben existir otros mecanismos de concentración y de adscripción diferencial mas allá de la antigüedad:

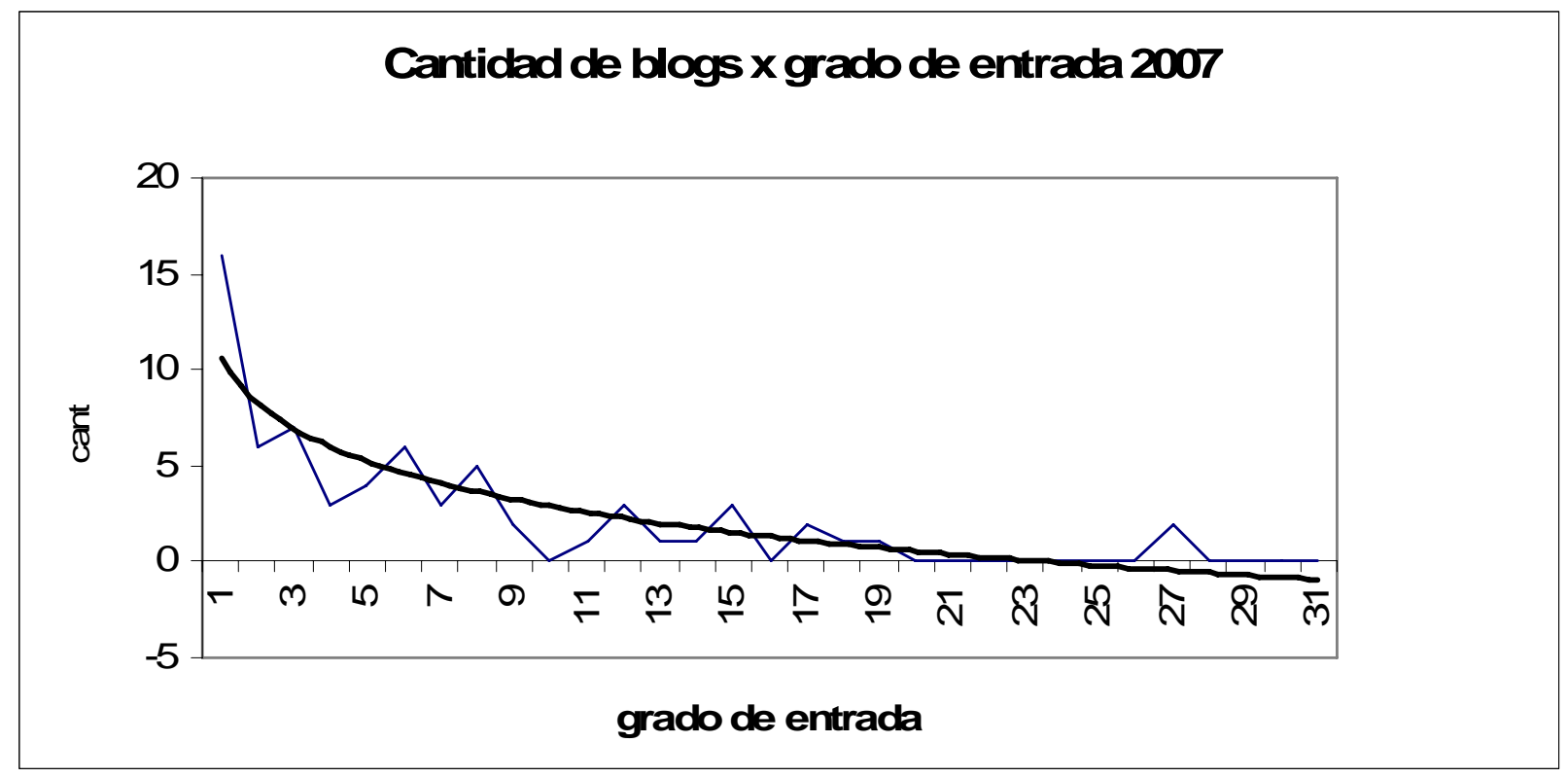

I lustración 7. Blogs por grado de entrada 2007. 


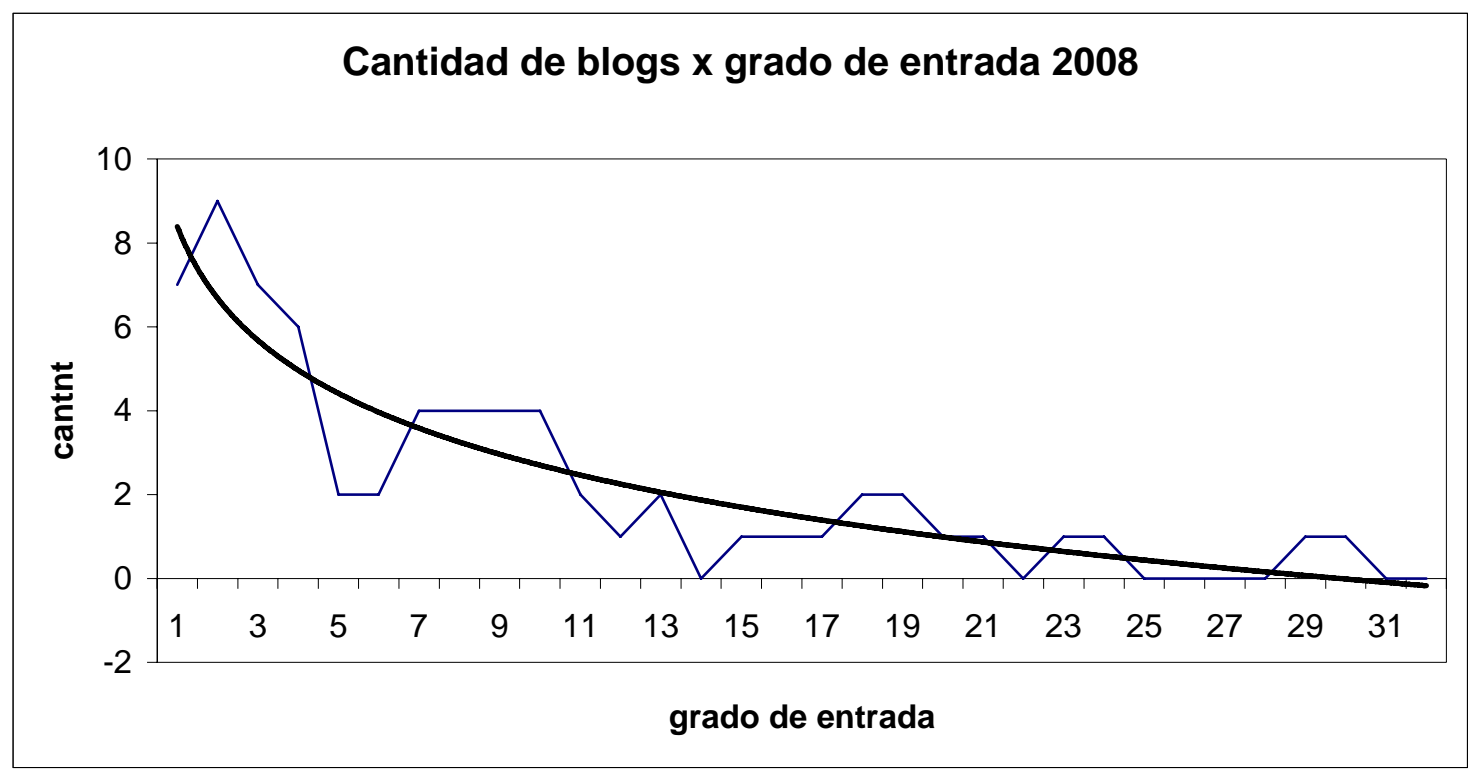

I lustración 8. Blogs por grado de entrada 2007.

Con una metodología similar a la que aplicamos en un trabajo anterior (Miceli y Guerrero 2007) vemos que la distribución del grado de entrada en los diferentes blogs tiene una distribución que se asemeja a la de las redes libres de escala, la distribución no tiene pico, como sí tiene la curva normal, y si bien es irregular (producto de el pequeño número de blogs seleccionados en relación a lo que es normal en este tipo de estudios), si la suavizamos se asemeja mucho a una función continuamente decreciente del tipo de las que representan las distribuciones libres correspondientes a los dos períodos considerados.

Parece un problema interesante para una investigación futura averiguar cómo se pudo haber producido este tipo de distribución con una diferencia tan pequeña en la antigüedad media entre los blogs del centro y de la periferia.

\section{Conclusiones}

El enfoque de redes en general, y las técnicas de análisis de redes sociales en particular, parecen adecuarse en gran manera al análisis de un fenómeno comunicativo y de posible creación de una comunidad sin un espacio físico o un sitio en línea común como es el de las redes de blogs y de la blogósfera.

Parece bastante promisorio en este campo hacer análisis que combinen lo reticular con lo atributivo, sobre todo si se quiere hablar del desarrollo y evolución de un nuevo ámbito de socialización e interacción social como es la blogosfera (o una parte de ella). 
Para poder explicar mejor su desarrollo esperamos en el futuro, comparar las estructuras mapeadas de esta red, correspondientes a 2007 y 2008 con otras, teniendo en cuenta no sólo los cambios en las relaciones sino también nuevos nodos que se agregan a la red.

También se pueden mapear las relaciones basadas en permalinks y comentarios cruzados, eso nos permitiría observar de manera más ajustada los cambios de la red en plazos más cortos, y nos permitiría formular hipótesis más precisas sobre el cómo y el porqué de su evolución.

Otro enfoque prometedor sería utilizar técnicas etnográficas tradicionales (Bernard 1995) o de etnografía virtual (Hine 2000), que puede que se ajuste mejor al tipo de interacciones que se da en este campo, con blogueros, comentaristas y lectores para intentar dar cuenta de sus prácticas y representaciones relacionadas con los blogs y las interacciones sociales generadas a partir de ellos. Con ello se puede complementar y enriquecer los resultados producto del análisis de redes sociales.

\section{Glosario}

Artículo (post): Cada una de las "entradas" que realiza el creador o administrador (bloguero) en su blog. También designadascomo apuntes o anotaciones pueden consistir en texto plano, imágenes o enlaces hipertextuales a otros posts o a contenidos de audio y/o vídeo para descarga o reproducciónBlog

Blog: También conocido como weblog o bitácora, es un sitio web actualizado de manera períodica que recopila con cronología inversa textos y/o artículos de uno o varios autores, con un uso o temática en particular, además conservando el autor la libertad de dejar publicado lo que crea conveniente.La mayoría de los blogs cuentan tambien con comentarios de lectores y un blogroll de otros sitios recomendados.

Blogosfera: Término usado para referirse a un conjunto de blogs, traducción del términoen inglés blogosphere. En ocasiones el concepto blogosfera se utiliza para referirseal conjunto de todos los blogs, en otras se utiliza para referirse a agrupaciones parciales de blogs, como por ejemplo la blogosfera argentina, la blogosfera política, etc. La blogosfera, en tanto que espacio de comunicación compartida, e incluso de comunidad, es resultadode la interconexión de los blogs a través de mecanismos como los hiperenlaces, los comentarios, etc.

Blogroll: Colección de hiperenlaces que publican los autores en su blog., generalmente en una columna a la izquierda o derecha de la página principal El blogroll suele estar compuesto por enlaces a otros blog, aunque no exclusivamente. El listado del blogroll está compuesto generalmente por blogs que el autor lee con asiduidad, que considerade especial interés o relevancia o que son de amigos o personas cercanas.

Comentarios: En cada artículo, designa las respuestas, sugerencias, opiniones y críticas de los lectores de un weblog. Constituyen el espacio donde tiene lugar la conversación pública entre el bloguero y sus lectores 
Hiperenlace: También llamado enlace, vínculo, hiperlink o hipervínculo. Es el mecanismo de referenciabásico en la World Wide Web. Un hiperenlace señala la dirección en la que se encuentra otro documento de hipertexto

Permalink: Enlaces permanentes en un weblog para que cualquiera pueda citar o recuperar una artículo. Como los nuevos artículos desplazan a los antiguos por el orden cronológico inverso, es importante facilitar un enlace que permita vincular a cada artículo con otros anteriores.

\section{Bibliografía}

Barabasi, Albert-Lazlo (2003). Linked. Nueva York: Plume.

Barabasi, Albert-Laszlo y Bonabeau Eric (2003). "Scale-Free Networks", Scientific American, Vol. 50.

Bernard, H.Russell (1995). Research Methods in Anthropology. Altamira Press Waiunut Creek California.

Borgatti, Steve, Everet Mg Freeman L.C. (2002). UCInet 6 Harvard MA. Analytic Technologies 2002.

Cameron, Marlow (2004). "Audience, structure and authority in the weblogCommunity" presenteda en la International Communication Association Conference, Mayo 2004, Nueva Orleans, LA. Disponible en http://alumni. media. mit.edu/ cameron/cv/pubs/04-01.html

Cameron, Marlow (2006). “Linking without thinking: Weblogs, readership and online social capital formation" Presentda en la International Communication Association Conference, June, 2006, Dresde, Alemania.

Drezner, Daniel y Farrell Henry. (2004). "The Power and Politics of Blogs". En www. utsc. utoronto.ca/ farrell/blogpaperfinal. pdf

Estalella Adolfo (2006). "La construcción de la blogosfera: yo soy mi blog (y sus conexiones)" en: José M. Cerezo. (2006). La blogosfera hispana: pioneros de la cultura digital. Fundación France Telecom España. Disponible en http://www.fundacionauna.com/areas/25_publicaciones/la_blogosfera_hispana.pdf

Goodman, Leo (1961). "Snowball Sampling". En Ann. Math. Statist. Volume 32, Number 1 (1961), 148-170.

Guerrero, Sergio (2007). “Aplicación del analisis de redes sociales de la estructura y desarrollo de una red de blogs económicos políticos en Argentina". Ponencia presentada en la ler. Reunión Latinoamericana de Análisis de Redes Sociales en La Plata, agosto de 2007

Hanneman, Robert A. Introducción a los métodos del análisis de redes sociales. Universidad de California Riverside. Versión en español en http://revistaredes.rediris.es/webredes/textos/

Herring, Susan et al (2005). "Conversations in the Blogpsphere: An Analysis from the Bottom Up" en Proceedings of the HICSS-38. Los Alamitos: IEEE Press. 
Hine, Christine (2000). Virtual ethnography, Londres: Sage.

Kumar, Ravi et al (2003). "On the Bursty Evolution of Blogspace" presentado en WWW 2003 Budapest Hungría mayo de 2003.

Merelo Juan, García Fernando, Pietro Beatriz (2004). “Visualización de la evolución de una red social generada por weblogs" Presentado en II congreso on line OCS.

Miceli Jorge y Guerrero Sergio (2007). "Redes libres de escala y su uso en el análisis de datos etnográficos: El caso de la comunidad tehuelche de Chalía" en Grupo Antropocaos Exploraciones en antropología y complejidad, Editorial SB Buenos Aires.

San Miguel, Maxi, Toral, Raúl y Eigueluz, Victor (2004). "Redes complejas en la dinámica social" en INGURUAK, Revista vasca de Sociología y Ciencia Política.

Sanz Menéndez Luis (2003). "Análisis de Redes Sociales: o como representar las estructuras sociales subyacentes" en Apuntes de Ciencia yTecnología N 7 junio de 2003.

Sifry David (2007). "The State of the Live Web," April 2007 disponible en http://www.sifry.com/alerts/archives/000493.html

Shirky Clay (2003). "Power Laws, Weblogs, and Inequality v.1.1" (2003) disponible en http://www.shirky.com/writings/powerlaw_weblog.html

Wasserman, Stanley y Faust Katherine (1994). Social Network Analysis: Methods and Applications. Cambridge University Press.

Watts Duncan (2003). Six Degrees. The Science of Connected Age. Random House, Londres. 